\title{
Multiwavelength radio observations of the compact starburst in Arp 220
}

\section{Rodrigo Parra $^{* a, f}$, John E. Conway ${ }^{a}$, Philip J. Diamond ${ }^{b}$, Hannah Thrall ${ }^{b}$, Colin J. Lonsdale ${ }^{c}$, Carol J. Lonsdale ${ }^{d}$ and Harding E. Smith ${ }^{e}$}

${ }^{a}$ Onsala Space Observatory, SE-439 92 Onsala, Sweden

${ }^{b}$ Jodrell Bank Observatory, Macclesfield, SK11 9DL, UK

${ }^{c}$ MIT Haystack Observatory, Westford MA 01886, USA

${ }^{d}$ Infrared Processing and Analysis Center, California Institute of Technology 10022 Pasadena CA 91125, USA

${ }^{e}$ Center for Astrophysics and Space Sciences, University of California, San Diego, La Jolla, CA 92093-0424, USA

${ }^{f}$ E-mail: rparralastro.puc.cl ${ }^{\dagger}$

\begin{abstract}
We report the first detection at multiple radio wavelengths $(13,6$, and $3.6 \mathrm{~cm})$ of 18 compact sources within both nuclei of the Ultra Luminous Infra-Red Galaxy (ULIRG) Arp 220. In just over half of the sources we find that the observed spectra are consistent with the standard model of powerful Type IIn supernovae interacting with their pre-explosion stellar wind. The rate of appearance of new radio sources ascribed to these supernova events suggests that a large fraction of core-collapse supernovae in Arp 220 are highly luminous, possibly implying a radically different stellar initial mass function (IMF) or stellar evolution compared to galactic disks. A second group of sources, consisting of the brightest and longest monitored sources at $18 \mathrm{~cm}$, do not easily fit the radio supernova model. We propose that these are young supernova remnants that have just begun interacting with their surrounding dense ISM.
\end{abstract}

8th European VLBI Network Symposium

September 26-29, 2006

Toruń, Poland

\footnotetext{
${ }^{*}$ Speaker.

${ }^{\dagger}$ Currently at Department of Astronomy and Astrophysics, Pontificia Universidad Católica, Chile
} 


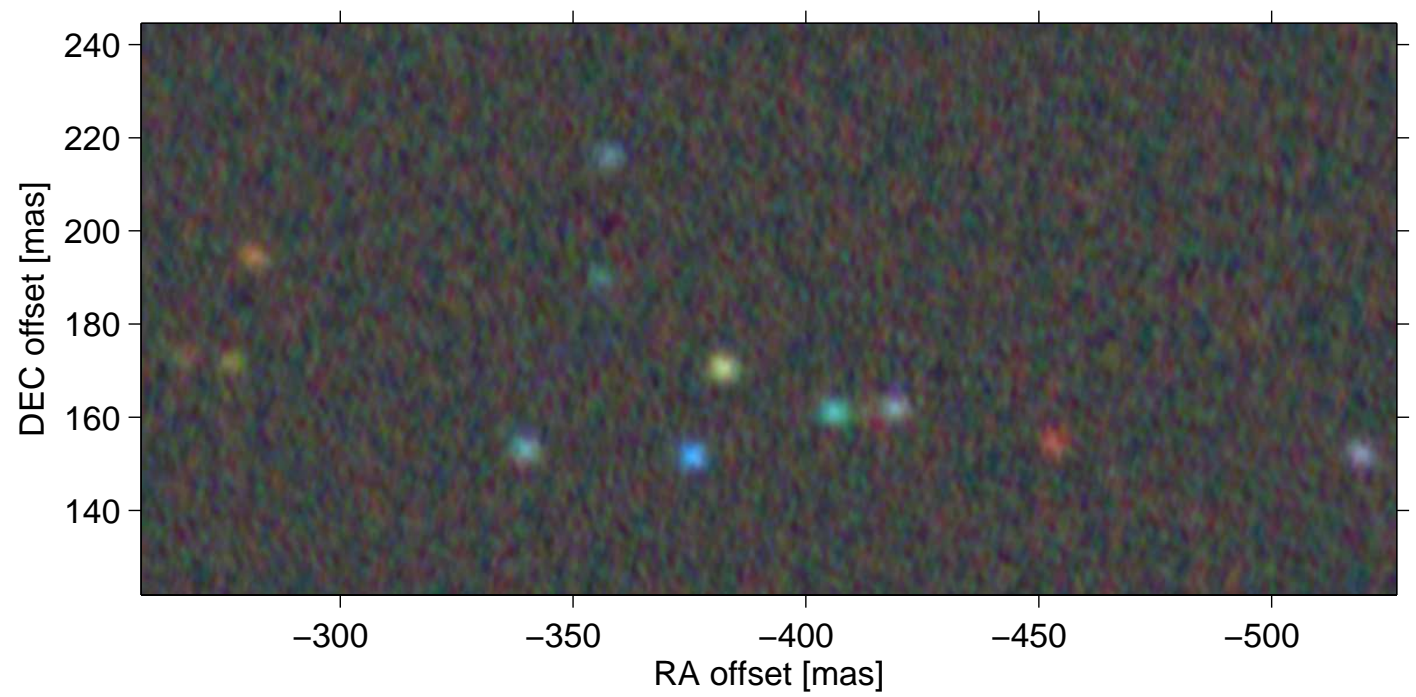

Figure 1: Detected compact radio sources in the western nucleus of Arp 220. This image combines the 13,6 and 3.6- $\mathrm{cm}$ maps represented in red, green and blue, respectively. The different colours demonstrate the wide variety of spectral properties of the sources. The axes are in mas from the reference position $\alpha_{2000}=15^{\mathrm{h}} 34^{\mathrm{m}} 57^{\mathrm{s}} \cdot 25, \delta_{2000}=+23^{\circ} 30^{\prime} 11^{\prime \prime} \cdot 33$.

\section{Observations and data reduction}

In order to map our EVN single-baseline 6-cm detections -see [3], we conducted VLBA ${ }^{1} 13$, 6, and 3.6-cm observations of Arp 220 during 10 hours on 9 Jan 2006. Instrumental delays and rates were removed by fringe fitting the bright calibrator $\mathrm{J} 1613+3412$ and applying the solutions to the data from the whole experiment. Atmospheric effects were removed by means of phase referencing with 2-minute scans on the nearby calibrator J1532+2344, alternating with 3.5-minute scans on Arp 220 at each wavelength. Amplitude calibration was based on system temperature measurements. Maps were produced at each wavelength using IMAGR and residual ripples were removed in a final stage using Fourier methods. The noise levels obtained at 13, 6 , and $3.6 \mathrm{~cm}$ are 130,86 , and $87 \mu \mathrm{Jy} \mathrm{beam}^{-1}$, respectively. A more detailed description of these observations can be found in [3].

\section{Spectra}

Figure 2 shows the spectra and model fits (see [3] for details) for the detected sources divided in two groups according to their variability at $18 \mathrm{~cm}$. The majority of the spectra are consistent with power-law synchrotron emission with turnovers below some critical frequency. Such spectra are typically observed in both supernovae (SNe) and supernova remnants (SNRs) [6, 1]

An important question is whether the compact radio sources in Arp 220 are primarily supernovae (interacting with their progenitors' circumstellar medium) or supernova remnants (interacting with the denser ISM). We find that for most of the sources showing variability at $18 \mathrm{~cm}$ (top panel of Figure 2) the fitted opacities and luminosities are in the range defined between the most luminous detected Type IIL SN 1979C and the Type IIn SN1986J. These sources are thus consistent with powerful SNe. On the other hand, it seems very difficult to explain the nature of the sources that are stable at $18 \mathrm{~cm}$ (bottom panel of Figure 2) using the same model. Although the diversity

\footnotetext{
${ }^{1}$ The National Radio Astronomy Observatory is a facility of the National Science Foundation operated under cooperative agreement by Associated Universities, Inc.
} 

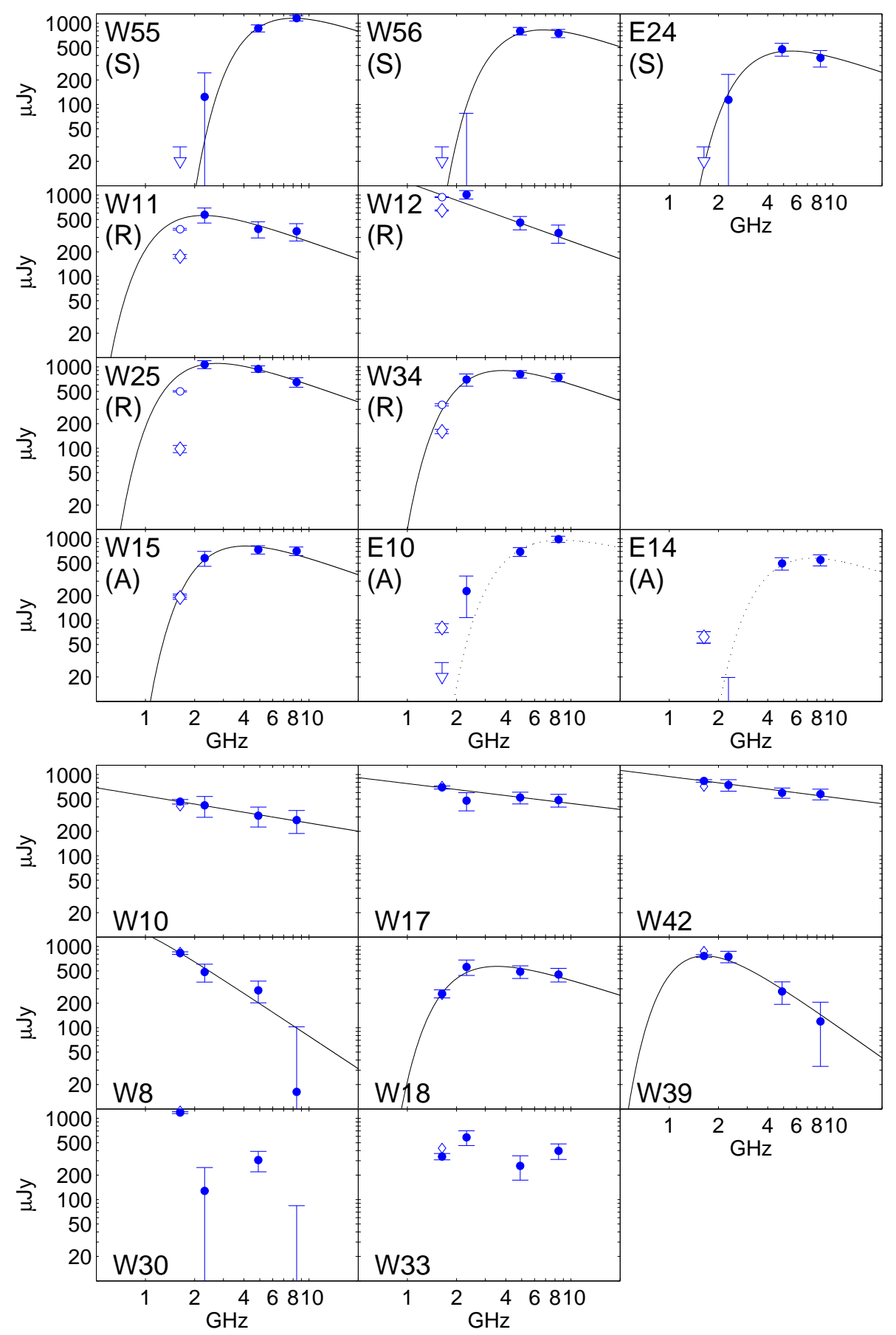

Figure 2: Top: Spectra and synchrotron plus free-free absorption models for the detected sources not in the original 18-cm sample of [5]. These sources are either new since 1995, weak at long wavelengths or both. Bottom: Spectra and synchrotron plus free-free absorption models for the detected sources in the original 18-cm sample of [5]. The first row shows three sources with relatively flat spectra. The middle row shows sources with steep spectra and the last row shows two sources for which good fits could not be obtained.

of their spectra is still a puzzle, we argue that, instead, these sources are SNRs in dense ISM environments. The proposed combined origin for the compact sources in Arp 220 offers a consistent explanation of the observed stability of the 18-cm light curves [4] without invoking SN events that 
are many times more luminous than the unusual SN1986J. If the rate of appearance of new radio sources is attributed to core-collapse $\mathrm{SN}$ explosions, then the required number of massive stars is much larger than predicted by the star-formation rate implying a top heavy initial mass function (IMF). This suggests that the initial mass function in Ultra Luminous Infra-Red Galaxies (ULIRGs) is perhaps radically different than in normal galaxies.

\section{Main conclusions}

1) For the first time we have detected the compact radio sources in Arp 220 at wavelengths shorter than $18 \mathrm{~cm}$. Previous failures to detect the compact sources at $6 \mathrm{~cm}$ appear to be largely due to using a phase calibrator that was too distant.

2) There is evidence that younger sources have higher free-free absorption, consistent with what is expected from RSN models.

3) The sources that are bright at $18 \mathrm{~cm}$, originally detected by [5], are best modelled by young SNRs interacting with the surrounding ISM. If they follow the same luminosity-size relation as the SNRs in M82, their luminosities imply diameters of $0.2 \mathrm{pc}$ and surrounding ISM of number densities in the order of $10^{4-5} \mathrm{~cm}^{-3}$. Ages for these sources would be only $30-50$ years, similar to those estimated by [2].

4) The rate of appearance of new radio sources approximately equals to that expected from the star formation rate if every core-collapse $\mathrm{SN}$ gives rise to a bright radio source [2]. However, slowly evolving SNe as bright as those detected in Arp 220 belong to the Type IIn class, which are thought to be relatively rare in galactic disks. Amongst the possible explanations to this apparent conflict are: that a totally different radio-SN paradigm applies to ULIRGs, that a top heavy stellar IMF or non-standard stellar evolution applies. A final possibility is that the recent starburst activity occurred in a very short but intense burst that we are now observing just as the most massive stars explode as $\mathrm{SNe}$.

\section{Acknowledgments}

RP acknowledges a Chalmers University PhD student stipend and JC a Swedish VR grant. This work was partially supported by NSF grant AST-0352953 to Haystack Observatory. The European VLBI Network is a joint facility of European, Chinese, South African and other radio astronomy institutes funded by their national research councils. The Arecibo Observatory is the principal facility of the National Astronomy and Ionosphere Center, which is operated by the Cornell University under a cooperative agreement with the National Science Foundation.

\section{References}

[1] Allen, M. L. \& Kronberg, P. P. 1998, ApJ, 502, 218

[2] Lonsdale, C. J., Diamond, P. J., Thrall, H., Smith, H. E., \& Lonsdale, C. J. 2006, ApJ, 647, 185

[3] Parra, R, Conway, J. E.,Diamond, P. J., Thrall, H.,Lonsdale, C. J.,Lonsdale, C. J.,\& Smith, H. E. 2006, ApJ, Submitted

[4] Rovilos, E., Diamond, P. J., Lonsdale, C. J., Lonsdale, C. J., \& Smith, H. E. 2003, MNRAS, 342, 373

[5] Smith, H. E., Lonsdale, C. J., Lonsdale, C. J., \& Diamond, P. J. 1998, ApJL, 493, L17+

[6] Weiler, K. W., Panagia, N., Montes, M. J., \& Sramek, R. A. 2002, ARAA, 40, 387 